\title{
Whitmore-Jewett Stage
}

National Cancer Institute

\section{Source}

National Cancer Institute. Whitmore-jewett Stage. NCI Thesaurus. Code C140161.

A prostate cancer stage defined according to the Whitmore-Jewett staging system criteria. 